\title{
An Approach to Hypertext Fiction for Mobile Devices
}

\author{
Matthew G. Styles \\ Brigham Young Univ. Hawaii \\ 55-220 Kulanui Street \\ Laie, Hawaii 96762 \\ mstyles@go.byuh.edu
}

\author{
Shin-Young Jung \\ Brigham Young University \\ 3361 TMCB \\ Provo, Utah 84602 \\ Idsrogan@byu.edu \\ Geoffrey M. Draper \\ Brigham Young Univ. Hawaii \\ 55-220 Kulanui Street \\ Laie, Hawaii 96762 \\ gmd2@byuh.edu
}

\author{
Chihiro Eto \\ Brigham Young Univ. Hawaii \\ 55-220 Kulanui Street \\ Laie, Hawaii 96762 \\ chihiro.eto@gmail.com
}

\begin{abstract}
Electronic literature has seen an explosion in popularity in recent years, due largely to the wide availability of smartphones, tablets, and dedicated e-reader devices. Somewhat surprisingly, mobile computing has been slow to embrace hypertext fiction. Yet the same qualities that make handheld devices popular for traditional linear narratives - small size, ease of use, and near ubiquity - also make them ideally suited for the distribution and consumption of hypertext narratives. In this paper, we review some existing systems for reading hypertext literature on mobile devices, and introduce Jarnaby Reader, a prototype e-reader for hypertext narratives that supports automatically generated overhead maps.
\end{abstract}

\section{Categories and Subject Descriptors}

H.5.4 [Hypertext / Hypermedia]: Theory; C.5.3 [Microcomputers]: Portable devices; J.5 [Arts and Humanities]: Literature

\section{General Terms}

Documentation, Design, Human Factors

\section{Keywords}

hypertext narrative, hypertext fiction, mobile computing

\section{INTRODUCTION}

Handheld computing devices — from the modest PDAs of the 1990s to the versatile smartphones and tablets of today - are undoubtedly one of the most important innovations in recent computing. With the increased popularity of handhelds, the public has likewise become increasingly accepting of the concept of electronic literature.

Permission to make digital or hard copies of all or part of this work for personal or classroom use is granted without fee provided that copies are not made or distributed for profit or commercial advantage and that copies bear this notice and the full citation on the first page. To copy otherwise, to republish, to post on servers or to redistribute to lists, requires prior specific permission and/or a fee.

NHT'12, June 25, 2012, Milwaukee, Wisconsin, USA.

Copyright 2012 ACM 978-1-4503-1408-4/12/06 ...\$10.00.
Handhelds present many advantages over traditional PCs for reading. First, they are portable; users can read e-books at the time and place of their convenience. Second, handheld devices typically boot faster than PCs, further increasing their convenience. However, these advantages alone do not explain the popularity of handheld e-book readers; after all, traditional books are likewise portable and "always on." Perhaps the greatest advantage of handheld e-books is their compactness relative to paper; a single mobile device, for example, can store the equivalent of an entire bookshelf. Stated another way, "Print stays itself; electronic text replaces itself."[14] This truth has not been lost on consumers, who have flocked to handheld devices in unprecedented numbers. Indeed, amazon.com recently reported that sales of their Kindle e-books outpaced sales of traditional printed books [1].

These developments have important implications for the hypertext community. After enjoying a burst of popular and academic attention during the 1980s and 1990s, interest in hypertext fiction has cooled somewhat in the past decade [3]. Although there are various reasons for this, the computing hardware of the time surely carries some of the blame. Most hypertext fiction titles were published when the desktop computer was the device on which most people spent the bulk of their computing time. Yet, although people willingly spend hours at a time in front of a desktop PC for work, gaming, or social networking, most people still do not consider their computers to be a "literary medium" [17].

Handheld computers have changed that. Although most readers may still lack the patience to sit down at a desktop PC to peruse an e-book, they nonetheless eagerly devour electronic literature when it is delivered wirelessly to their smartphone or tablet. The battle for electronic literature has been won - thanks to a myriad of handheld computing devices. ${ }^{1}$ If handheld devices have proven themselves as a suitable medium for reading traditional linear narratives, would they not be doubly effective for navigating nonlinear hypertext narratives? It follows that readers who may have historically been reluctant to try hypertext fiction may re-

\footnotetext{
${ }^{1}$ Handheld computers also render obsolete the infamous "Bolter Test" [4, 7], which states that, to be widely accepted, electronic literature must be readable in bed or in the bath. With an inexpensive waterproof case, users can take their handheld devices not only to the bath, but to the beach or swimming pool.
} 
consider it if presented on a more suitable medium, such as a handheld device. We feel that the technology has finally caught up to the genre, and thus, the time may be right for a renewal of popular and critical interest in hypertext fiction.

The remainder of the paper is organized as follows. We provide an overview of hypertext as a literary form in Section 2 . In Section 3 we describe early attempts at migrating non-linear forms of narrative to handheld devices. In Section 4 we introduce our contribution to the field, Jarnaby Reader, a prototype system for viewing multi-perspective hypertext narratives on handheld devices. Finally, we draw some conclusions and offer possible avenues for future work.

\section{HYPERTEXT NARRATIVE}

Although many of our readers are likely already familiar with the area of hypertext fiction, for completeness we provide a brief review of the genre. Hypertext has a long and venerable history as a medium for electronic storytelling. Canonical works of hypertext fiction include afternoon, a story by Michael Joyce and Patchwork Girl by Shelley Jackson, both published by Eastgate Systems [10].

When introducing hypertext fiction to those unfamiliar with it, there is a temptation to compare it to the Choose Your Own Adventure novels that were popular in the late 20th century. Although these books are non-linear and therefore somewhat hypertextual, this comparison may confuse more than it illuminates. Indeed, hypertext fiction in general "seldom invites or permits the reader to select actions for the hero protagonist." [4]

Reading a hypertext novel is different from reading a traditional linear printed narrative. A single page of text (called a lexia [2], usually no more than a few paragraphs) is displayed on the screen, and the user advances the narrative by selecting one of potentially many hyperlinks embedded in the text. Which lexia the reader encounters next depends on which link is followed. Although a default link is typically provided which yields a somewhat linear reading, other links may, for example, take the reader to a different time in the story, to a different character's perspective, to a different location in the fictional world, or simply provide background information on a particular word, location, or character.

Since the author of a hypertext fiction cannot know in what order the reader will traverse the links, the story may take on many different forms. It is only through repeated readings, by revisiting lexias under different contexts, that the fragments of text gradually coalesce, allowing the reader to assemble his or her own idea of the events of the story [5].

\section{NON-LINEAR ELECTRONIC LITERATURE FOR HANDHELDS}

Hypertext fiction published on the Web [11] is, of course, available on any handheld computer with a browser and an Internet connection. However, the limitations of the Web for publishing effective hypertext fiction are well-known [5, 11]. Moreover, certain technologies that enrich a browser's capabilities, such as Java, JavaScript, and Flash, have limited or no support on many mobile devices. Finally, the fonts and layout of a web page designed for desktop viewing may not transfer well to small screens (see Figure 1).

For these reasons, we believe the future of electronic literature on handhelds lies not within the browser, but in apps developed specifically for reading non-linear narratives.

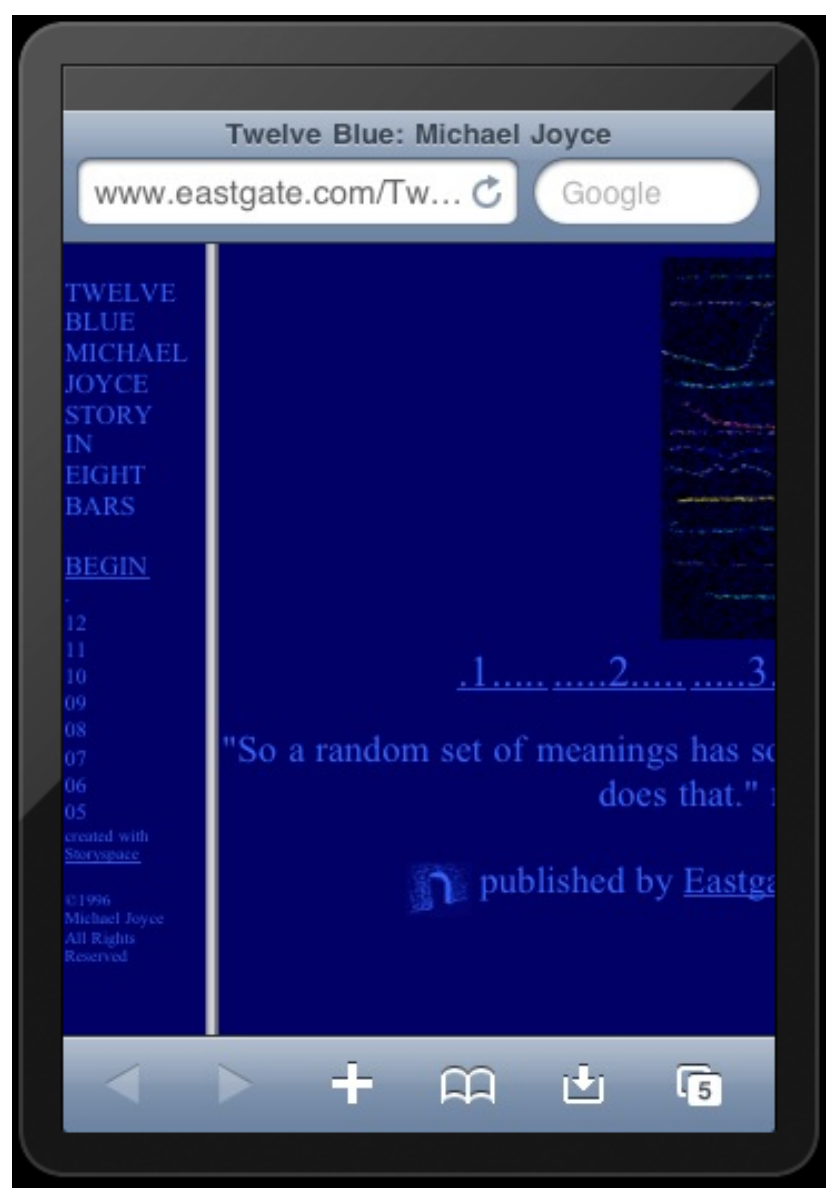

Figure 1: Michael Joyce's online hypertext Twelve Blue as rendered in the iPhone's Safari browser. Notice that the page layout was designed for large screens, making navigation somewhat cumbersome on a handheld device. (c)1996 Michael Joyce.

\subsection{Legacy Works Revitalized}

While we have seen relatively few attempts to create new non-linear narratives for handhelds, there has been some significant efforts to breathe new life into legacy titles by porting them to modern platforms [9].

\subsubsection{Interactive Fiction}

Ironically, it is interactive fiction (IF), rather than hypertext fiction, that was first to embrace handheld computing. ${ }^{2}$ Two apps in particular, Twisty (for Android) and Frotz (for iOS) are free IF readers that can play nearly any of Infocom's old commercial titles or the more recent noncommercial ones. The popularity of IF on handheld devices is surprising, considering the difficulty of entering lengthy commands onto a miniature keyboard. Given that a typical hypertext fiction story requires little or no keyboard input

\footnotetext{
${ }^{2}$ Also known as "text adventure," IF is a genre of electronic literature that is sometimes compared to hypertext fiction. IF enjoyed a heyday of commercial success in the 1980s under the leadership of Infocom, Inc. (later acquired by Activision). Today, IF is still being produced - albeit noncommercially - and has a small but devoted grassroots following [17].
} 


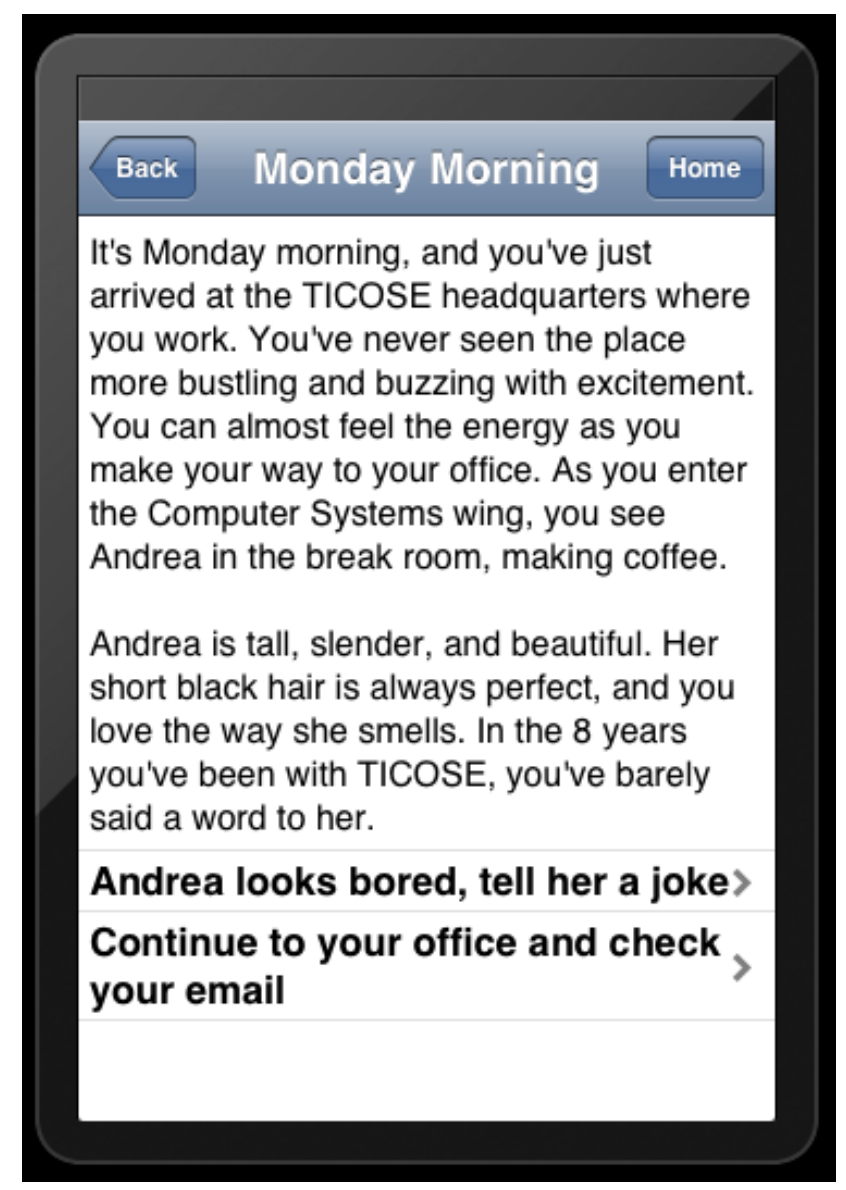

Figure 2: iStory's hyperlinks are clearly labeled, and consistently positioned at the bottom of each lexia. This screenshot is taken from "Mission to the Moon," which is included as one of the default titles in iStory. (C) 2009 Taylor Steil

on the part of the user, we believe hypertext fiction is an even better fit for handhelds than IF is.

\subsubsection{HyperCard-based Works}

In the 1980s and 1990s, HyperCard was a tremendously popular and influential system for creating hypertext documents. So versatile was this tool that some developers used it to create graphical virtual environments. In the commercial arena, perhaps none were more successful than Cyan Worlds, whose HyperCard-based games The Manhole and Myst achieved popular and critical acclaim. Myst in particular spawned a number of sequels and is among the most beloved computer games of all time [18]. These games were more hypermedia than hypertext, the "links" being hotspots on the screen that the user could activate to explore additional locations in the simulated world. Beginning in 2009, Cyan Worlds ported The Manhole, Myst, and its sequel Riven to the iPhone, where these titles have achieved a second wave of success.

\subsection{Hypertext Fiction for Beginners}

An iPhone app for reading hypertext fiction, iStory [12] can be used to read both traditional narratives as well as hypertexts. Like conventional hypertext fiction systems, iStory presents the reader with a series of lexias, one screen at a time. However, iStory employs a simplified user interface that disallows hyperlinks within the body of a lexia. Instead, links are placed predictably at the bottom of the screen, and allow the reader to follow one of potentially many discrete paths through the story (see Figure 2). This is, by design, a similar convention to that used by the Choose Your Own Adventure novels, thus virtually eliminating the learning curve to iStory for anyone familiar with these books.

$i$ Story comes bundled with approximately 20 stories, ranging from simple adventures to mysteries to light comedies. StoryForge, a companion piece of software to iStory, allows authors to create their own hypertext stories and distribute them through iStory.

\section{JARNABY READER}

We believe that iStory goes a long way towards bringing hypertext fiction to handheld devices, and we applaud it. However, Jarnaby Reader (see Figure 3) addresses two issues that are not entirely resolved by iStory.

First, while iStory's placement of the links at the end of each lexia simplifies the user interface, it also encourages the development of "quest"-style stories where the reader directs the actions of the protagonist. While that is one valid application of hypertext fiction, it is certainly not the only one.

Second and more importantly, we wanted to alleviate user disorientation, a common experience for those experiencing hypertext fiction for the first time [20]. Thus, Jarnaby Reader supports dynamically-generated overhead maps, which allow readers to visualize "where they are" in the story, relative to previously-visited lexias.

Finally, we wanted our system to be cross-platform, which is why we have made Jarnaby Reader available for iOS, Android, and BlackBerry. ${ }^{3}$ Jarnaby Reader is released under a free software license.

Like iStory, Jarnaby Reader uses a simplified user interface where hyperlinks are clearly marked and predictably located. However, Jarnaby Reader is best suited for reading "multi-character" stories. Like any hypertext fiction system, the entire narrative is divided into a series of discrete lexias. In Jarnaby Reader, the lexias are further grouped into paths. A path is the sequence of events as experienced by any one character in the story. Thus, there is one path for every principal character in the narrative. In some stories, the characters' paths may coincide much of the time; in others, their paths may be largely disjoint. The number of paths and the degree to which they intersect depend, of course, on the story.

As a proof of concept, Jarnaby Reader comes bundled with a sample hypertext story based on the traditional fairy tale Little Red Riding Hood [5]. The default path through the story follows the perspective of the eponymous main character. Other paths exist for the other main characters in the story; the reader can switch to these paths at key locations within the narrative.

Each lexia contains a button labeled Next which acts as a "default hyperlink" which takes the reader to the next logical lexia in the current character's path. The Pre button serves

\footnotetext{
${ }^{3}$ For downloads and documentation, please refer to www.jarnaby.com.
} 


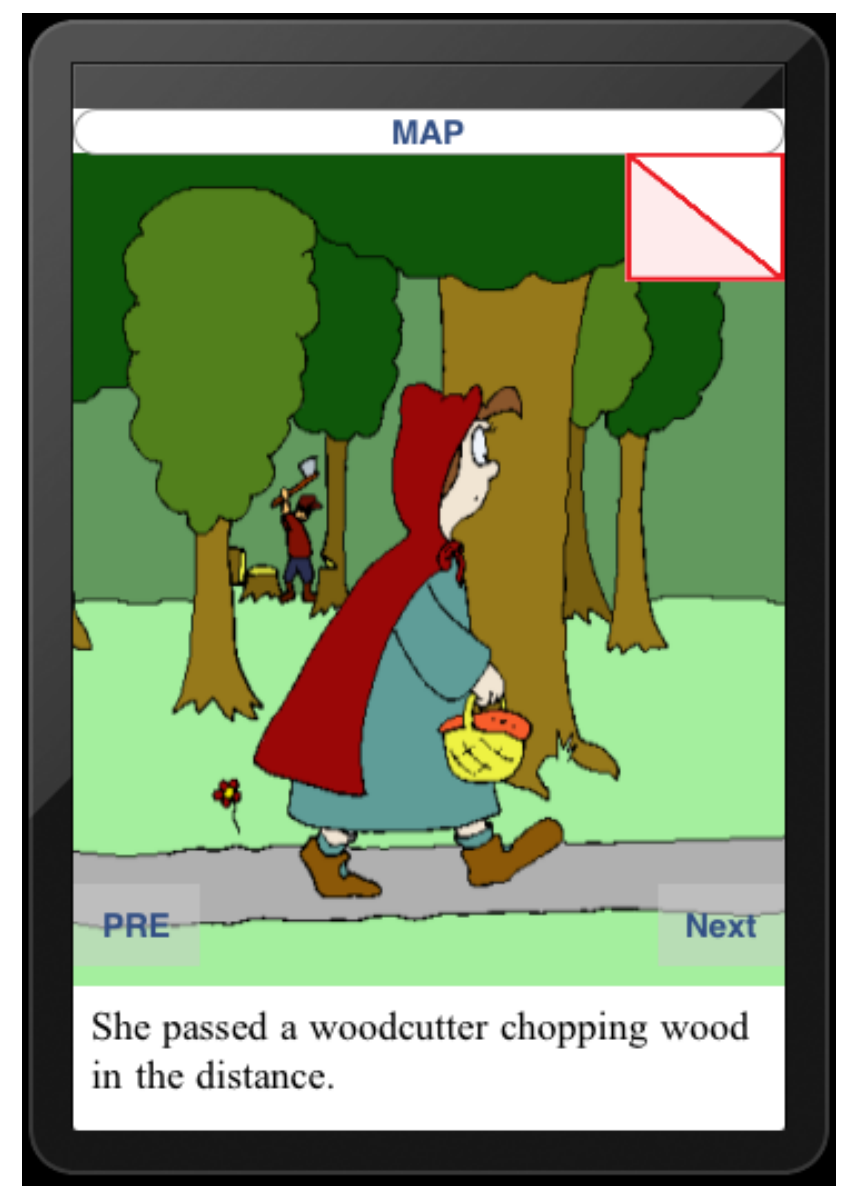

Figure 3: Jarnaby Reader is best suited for multicharacter stories. Although a story can be read linearly, the system allows the reader to switch to other characters' paths at key locations in the narrative.

as a "backlink" to the previous lexia in the current character's path; or, if the current lexia was reached by following a hyperlink from another character's path, the Pre button restores the reader to the former path. The presence of the "dog ear" in the top-right corner of Figure 3 means that this particular lexia contains a link to another character's path. ${ }^{4}$ The reader may activate the hyperlink either by tapping the "dog ear" or by tapping the on-screen character whose point of view the reader wishes to follow. The transition [16] from one perspective to another is marked by a brief animation (in the iOS version) or an unobtrusive white flash (in the Android and BlackBerry versions).

While it is perfectly acceptable to read the entire story from the point of view of the default character, as the reader examines additional characters' paths, he or she begins to see how each separate subplot interweaves to construct the whole story. When the reader finishes one character's path,

\footnotetext{
${ }^{4}$ Jarnaby Reader's placement of hyperlinks in the corner of the screen was inspired by Infocomics, a series of hypertext ebooks published by Infocom, Inc. just prior to its acquisition by Activision. Infocomics was a notable experimentation with hypertext by a company that is remembered chiefly for its contributions to interactive fiction.
}

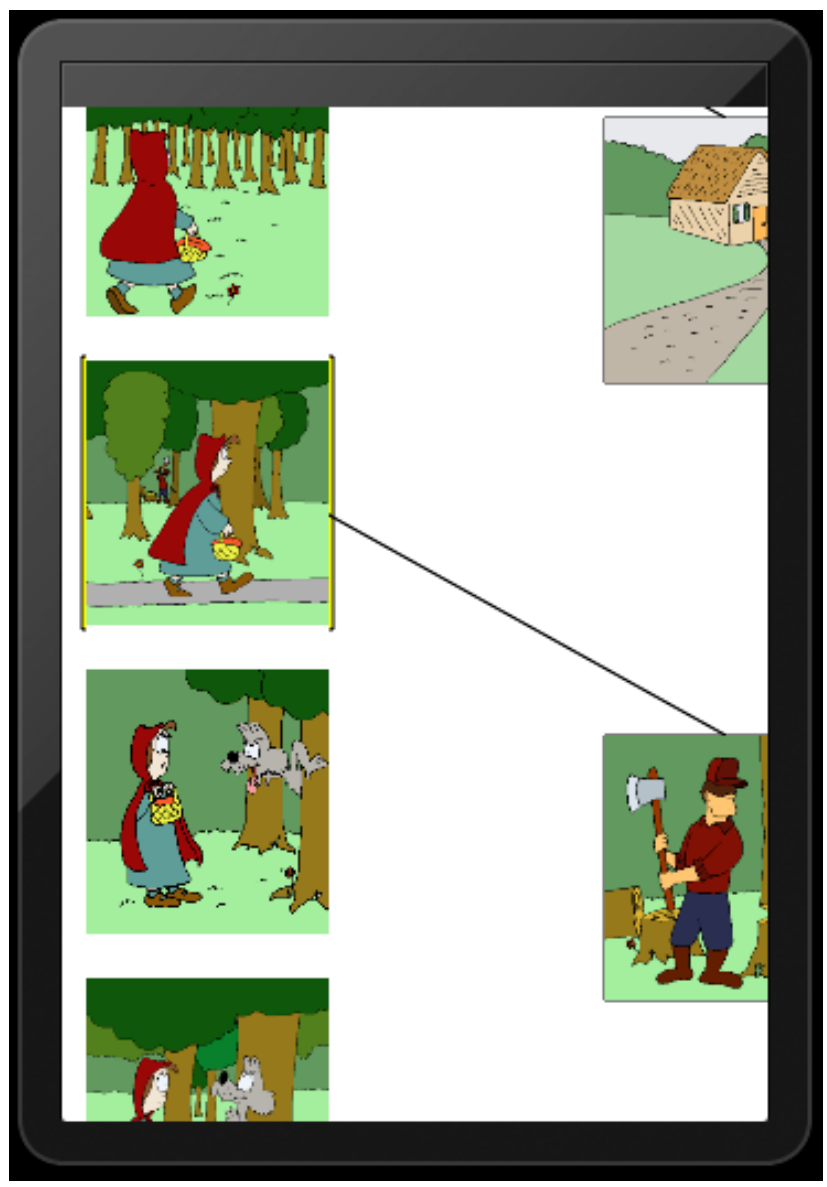

Figure 4: Jarnaby Reader's interactive "overhead map" lets the reader visualize how the hyperlinks connect the various lexias in the story.

the system gives the reader the option to return back to the story to view as-yet unexplored narrative paths.

At the top of the screenshot in Figure 3, there is a button labeled Map. Tapping this button opens up a birds-eye view of the current path in the story (see Figure 4). Each lexia is represented as a node in a graph, and hyperlinks between the current path and other characters' paths are represented as edges. As shown in the figure, each node is rendered as a thumbnail icon of the lexia's artwork. Currently unvisited nodes are decorated instead with a question-mark icon. The user can pan around the map by swiping the screen with his or her finger. Tapping on a node takes the reader to the corresponding lexia in the story, exiting map mode. While overhead maps have been employed in previous hypertext systems [8], these maps are typically crafted by hand. In contrast, the maps used in Jarnaby Reader are generated automatically by the software, based on an XML description of the story provided by the author.

\section{DISCUSSION AND FUTURE WORK}

In this paper, we argue that the ubiquity of handheld computing devices, along with society's acceptance of these devices as a means of distributing and enjoying electronic literature, presents a unique opportunity for authors and publishers of hypertext fiction. We have reviewed some existing 
systems for viewing non-linear narratives on handhelds, including our own prototype system, Jarnaby Reader.

As a work in progress, Jarnaby has several avenues of further research. First, we would like to conduct a user study to evaluate [6] the usefulness of the overhead maps for reducing readers' disorientation. A possible test might be to have a two groups of participants read the same story, giving one group access to the overhead maps but not the other. Measurements could include overall time spent reading the story, total number of nodes visited, and frequency of switching from one character's path to another. This could be followed up with a qualitative survey to measure the reader's satisfaction with the interface.

Another opportunity for future work concerns Jarnaby Reader's XML file format. Although not overly complex, XML may not be an author's first choice for writing fiction, hypertext or otherwise. As a future work, we would like to develop a complementary "Jarnaby Writer" system that provides authors a more intuitive environment for constructing multi-character stories. Alternatively, plugins could be built for existing tools such as Storyspace [19], to output story files compatible with Jarnaby Reader. Either way, we believe that systems for authoring hypertext fiction will likely remain on the desktop, rather than on mobile devices. In contrast to reading (which can be done anywhere), most creative writing is still best done on large screens.

Additionally, we would like to explore other ways to use hyperlinks in a story. While Jarnaby currently supports links between characters, some narratives might require links from one object (not necessarily a character) to another, or across different moments in time. Furthermore, the "mobile" aspect of Jarnaby could be further embellished by using the device's GPS capabilities to customize the narrative based on the reader's current location in the world [13].

Although we hope that Jarnaby Reader will be an important node in the conversation, it is not the final answer to hypertext fiction on handhelds. Jarnaby Reader's primary contributions are its streamlined interface for viewing a story from different characters' perspectives, and its novel use of automatically generated overhead maps to decrease user disorientation. However, it does not yet support advanced features such as guard fields [15] or video, as found in professional (desktop-based) hypertext tools. Therein lies the most exciting area of future work: to develop handheld hypertext e-reader systems with similar capabilities as their desktop counterparts, thus enabling a new generation of hypertext storytelling.

\section{REFERENCES}

[1] BBC News. Amazon kindle e-book downloads outsell paperbacks. http://www.bbc.co.uk/news/business-12305015, 2011. Accessed 24 January 2012.

[2] A. Bell. The Possible Worlds of Hypertext Fiction. Palgrave Macmillan, Hampshire, England, 2010.

[3] M. Bernstein. About tekka: A note from the publisher. http://www.tekka.net/Tekka/About.html, 2003. Accessed 18 January 2012.

[4] M. Bernstein. Into the weeds. In M. Bernstein and D. Greco, editors, Reading Hypertext. Eastgate Systems, 2009.

[5] M. Bernstein. On hypertext narrative. In Proceedings of the 20th ACM conference on Hypertext and hypermedia, HT '09, pages 5-14, New York, NY, USA, 2009. ACM.

[6] M. Bernstein. Criticism. In Proceedings of the 21st ACM conference on Hypertext and hypermedia, HT '10, pages 235-244, New York, NY, USA, 2010. ACM.

[7] J. D. Bolter. Writing Space: Computers, Hypertext, and the Remediation of Print. Routledge, London, 2001.

[8] D. Ciccoricco. Returning in twilight: Joyce's twilight, a symphony. In M. Bernstein and D. Greco, editors, Reading Hypertext. Eastgate Systems, 2009.

[9] G. M. Draper. The art of rewriting old games. Linux Journal, October 2003.

[10] Eastgate Systems, Inc. http://www.eastgate.com. Accessed 18 January 2012.

[11] Eastgate Systems, Inc. Hypertext reading room. http://www.eastgate.com/ReadingRoom.html. Accessed 24 January 2012.

[12] iStory. http://istoryweb.appspot.com/.

[13] M. Jewell and C. Hooper. Linking locations: Storytelling with pervasive technology. In HYPERTEXT 2011: Workshop on Narrative and Hypertext. ACM, 2011.

[14] M. Joyce. Of Two Minds: Hypertext Pedagogy and Poetics. University of Michigan Press, Ann Arbor, Michigan, 1996.

[15] M. Joyce. Nonce upon some times: Rereading hypertext fiction. In M. Bernstein and D. Greco, editors, Reading Hypertext. Eastgate Systems, 2009.

[16] C. Mancini. From cinematographic to hypertext narrative. In Proceedings of the eleventh ACM on Hypertext and hypermedia, HYPERTEXT '00, pages 236-237, New York, NY, USA, 2000. ACM.

[17] N. Montfort. Twisty Little Passages: An Approach to Interactive Fiction. MIT Press, Cambridge, Massachusetts, 2003.

[18] J. Parish. The essential 50: Myst. http://www.1up.com/features/essential-50-myst, 2004. Accessed 18 January 2012.

[19] Storyspace. http://www.eastgate.com/storyspace/.

[20] J. Walker. Piecing together and tearing apart: finding the story in afternoon. In Proceedings of the tenth ACM Conference on Hypertext and hypermedia, HYPERTEXT '99, pages 111-117, New York, NY, USA, 1999. ACM. 